\title{
Optimization of urban bus operation frequency under common route condition with rail transit
}

(C) The Author(s) 2017. Published by Higher Education Press. This is an open access article under the CC BY license (http:// creativecommons.org/licenses/by/4.0)

\begin{abstract}
The overlap of bus and rail transit routes is common in China. This overlap provides passengers multiple choices for one trip. However, the availability of multiple options would cause uncertainty in the travel distribution of passengers. Given that buses and rail transits are becoming increasingly common, this paper aims to present the overlapped operation condition of bus and rail transit using a bi-level model from the perspective of bus operators. Frequency optimization model is established in the upper-level model. A heuristic algorithm called shuffled complex evolution (SCE-UA) method is used to solve the established frequency optimization model, and three other heuristic methods are compared with SCE-UA. A lower-level Logit model based on Agent simulation is set for traffic mode split. Data on the transit system in Dalian city are chosen as an example to test the feasibility of the model and the algorithm. Results show that as the overlapped optimization of bus route and rail transit routes changed primary bus frequency, the use of SCE-UA to solve such problems has evident advantages and feasibility; furthermore, changed bus frequency would improve bus operations.
\end{abstract}

Keywords common route, bus operation frequency, bilevel model, Agent simulation, SCE-UA algorithm

\section{Introduction}

Rail transit operation will break the balance of the existing

Received May 2, 2017; accepted September 4, 2017

Bin YU, Sijia REN, Enze WU, Yunpeng WANG ( $\triangle)$

School of Transportation Science and Engineering, Beihang University, Beijing 100191, China

E-mail: ypwang@buaa.edu.cn

Yifan ZHOU

Transportation Management College, Dalian Maritime University, Dalian 116026, China traffic mode choices of passengers in terms of service (Verma and Dhingra, 2006). On the one hand, some passengers opt for rail transit, which will reduce the number of passengers traveling by bus. Thus, ensuring the frequency of bus operation is a challenge. On the other hand, rail transit cannot guarantee that it can simultaneously carry enough passengers, which will cause massive waste of public investments. Therefore, the availability of multiple choices on urban bus and rail transit to passengers has become a pertinent issue.

This paper proposes optimizing the conditions of bus and rail operations by using a bi-level model. Based on traffic flow characteristics, conventional buses are for short-distance transport, whereas rail transit is usually for long-distance transport. Mohaymany and Gholami (2010) identified different levels and functions of passenger transport systems. Although bus and rail transit have several investment differences, such as traffic capacity, speed, and cost, the two still compete. This study analyzes passengers' choices between bus and rail transit using a traffic mode split model. The model can determine a passenger's choice in each traffic mode and effectively maintain bus operations under common rail transit route conditions.

In the traffic mode split model, the share rate of passengers is an important research field. This model is specifically used to study passengers' choices. Numerous studies have focused on passengers' choices, and they can be divided into three based on the methods adopted. One is disaggregate choice model based on individual choice behavior, and the others are distribution and neural network models, which are based on network traffic. Various methods have different characteristics when determining the influence factors of utility function and specific model forms. Mandel et al. (1992) proposed the share rate model of bus and rail using Logit model under the condition of a high-speed rail going into operation. When comparing the precision of models in terms of linear and nonlinear utility functions, the nonlinear utility 
function model was found to have better explanatory power. Nijkamp et al. (1996) compared the choice probabilities of bus and rail traffic modes by fitting different origin-destination (OD) pairs between neural network and Logit model. Nuzzolo et al. (2000) evaluated the rail transit supply-demand and fare policy with the utility function indicating the expected arrival time and early (late) time of delay. The model was established based on Nested-Logit (NL) which considered service, frequency, class, and accessibility. Ben-Akiva and Morikawa (2002) studied whether passengers prefer rail transit over bus. Results showed that passengers have no evident preference when keeping the same service. However, passengers prefer an improvement in rail transit service. Yao et al. (2014) presented an optimization model, which considered stochastic travel time to maximize the efficiency of passengers' trips in the transit network. Tabu search algorithm was then used to solve the optimized transit network. Results showed the negative correlation between travel time and cost, whereas service level was positively correlated with selective probability. GonzálezSavignat (2004) used the survey data from an experiment to establish a Binomial-Logit model according to travel purpose and distance. Travel cost, time, bus frequency, energy consumption, and charge were used as model variables. Findings indicated that the main factors were travel cost and time when bus frequency was reduced. Yu et al. (2011) proposed models to predict bus arrival time at the same bus station but with different routes; they adopted several methods, including support vector machine, artificial neural network, k-nearest neighbors algorithm, and linear regression.

Bus frequency optimization is a major component of transit operation. This paper focuses on the perspective of bus operators who aim to maximize profit. An optimal model for the bus frequency of a bus route is developed to maximize the total profit of bus operators.

Several studies have focused on optimizing bus frequency. Salzborn $(1972,1980)$ proposed simplified mathematical models for designing frequency to minimize bus fleet and passengers' waiting time. Furth and Wilson (1981) devised a mathematical method for optimizing the allocation of buses to routes and maximizing net social benefit; they proposed an algorithm based on Kuhn-Tucker conditions. Han and Wilson (1982) proposed a two-stage heuristic algorithm for allocating vehicles to routes: in the first stage, a minimal frequency that can satisfy the demand is determined, and in the second stage, frequency is increased uniformly until all vehicles of the available fleet are used while considering constraints on total fleet size and route capacities. LeBlanc (1988) formulated a transit network design model for determining transit frequency under the assumption of elastic modal split and proposed to solve the model by Hooke-Jeeves algorithm (HJA). Constantin and Florian (1995) developed a mixed-integer programming model and proposed a projected subgradient algorithm for optimizing frequency to minimize passengers' total travel and waiting time. they proposed a projected sub-gradient algorithm for solving the problem. Tom and Mohan (2003) presented a model to optimize transit routes and frequencies on the basis of genetic algorithm (GA) to minimize operation costs and passengers' total travel time. Park (2005) used a simulation-based GA to optimize headways and slack time. Chakroborty (2003) and Chakroborty et al. $(1995,2001)$ utilized a GA to solve a bus-scheduling problem. Their results showed that GA was an efficient solution tool for several optimization problems in the transport field. Yu et al. (2010) proposed a GA to solve a bi-level optimization problem on bus frequency, which aims to minimize the passengers' total travel time subjected to the constraint of overall fleet size. Blum and Mahew (2011)reported that bus transit route network optimization emphasizes bus routes and schedules. Passenger demand was satisfied, and bus company cost was lowest when bus routes and schedules were optimized. Baaj and Mahmassani (1995) designed a route-generation algorithm to optimize bus routes and frequency. Agrawal and Mathew (2004) developed an optimization model of bus transit route network, which aims to minimize transport costs, and calculated the model using GA. Kuan et al. (2006) proposed GA and ant colony optimization (ACO) to solve the problem of bus frequency optimization on the basis of passenger competition and collaborative analysis.

Given that the optimal model addresses a bus scheduling problem, its practical application is complicated by increasing spaces, roads, and stations. Numerous studies have shown that the heuristic algorithm can effectively solve complex combinatorial optimization problems. Thus, the shuffled complex evolution (SCE-UA) algorithm (Duan et al., 1992), which has been successfully used in the field of water resource, is adopted. The algorithm is based on a simplex method with a random search, biological evolution principles, and complex blends (Duan et al., 1992; Duan et al.,1993) integrated together.

With reference to existing studies, the main contributions of this paper are as follows:

(1) This study explores the bus frequency optimization problem in terms of bus operators' profit and proposes a bilevel model. In the upper-level model, an objective model of bus and rail competition is established, which considers bus operator interest and operating costs. The lower-level model is the traffic model split by using the Logit model, which considers factors such as waiting time, travel time, and crowding, among others. This paper presents an effective method to solve the bus frequency optimization problem.

(2) The SCE-UA is proposed to solve the same problem. Three other commonly used methods, namely, GA, HJA, and ACO are compared with SCE-UA. After simulations 
and calculations, a balance point, which maximizes bus operators' profit, is derived. The Dalian-Kaifaqu (D-K) bus route and Light Rail Route No. 3 of Dalian city are chosen as examples to test the model. Computational results suggest the effectiveness and evident advantages of SCE-UA.

This paper is organized as follows. Section 2 describes the problems. Section 3 proposes a bi-level model. Section 4 introduces SCE-UA. In Section 5, the data of Dalian city is chosen as an example to test the model. The last section concludes the paper.

\section{Problem description}

In the public transit network, the stations of different lines are usually located close to one another. This condition is called transit route overlap. Therefore, transit stations also overlap, which means that stations of different vehicles are also located closely. In Fig. 1, the black and blue lines represent the bus and rail routes, respectively. In this figure, bus and rail routes overlapped in the first four stations, separated in the fifth station, and overlapped in the sixth and seventh stations. If the bus and rail routes do not overlap, passengers could select only one route in a trip. However, if two stations overlap, passengers will consider factors such as travel time, travel fare, and congestion in the two transport means in choosing their travel routes. The model of two transport means should adopt traffic mode split by using a Binomial-Logit model. When the frequency of bus changes, it will produce extra operating fare. Bus frequency will also change the operator's total revenue. From the bus operators' perspective, this paper proposes a bus frequency model based on routes to maximize total profit.

\section{Competition model of bus and rail transport}

\subsection{Factors of bus and rail transit options}

When passengers choose transport means, they will not only consider individual characteristics but also the characteristics of different traffic modes (Nakagawa and Hatoko, 2007). The specific factors are as follows:

(1) Travel time. This factor generally refers to the time taken to complete one travel. Passengers usually prefer to choose a time-saving and convenient traffic mode, and travel time becomes an important factor for evaluating different transport modes.

(2) Travel fare. Travel time savings are usually paid for by higher fare costs. So travel fare and travel time are generally considered.

(3) Congestion. The comfort level for passengers during travel becomes increasingly high with the improvement of living standards. Passengers always desire to have a pleasant trip.

(4) Security and reliability. Passengers constantly desire sufficient security during a trip because of the accelerated pace of life. Passengers have a high demand for travel punctuality because they have a stronger sense of time.

The factors affecting the traffic mode of passengers include travel time, fare, congestion, security, and reliability. However, according to Lam et al. (1999), the most important factors are travel time, fare, and congestion. Therefore, these three are selected as the main factors in this study. Considering that security and reliability are hard to quantify, this paper adopts a fixed parameter to express these factors.

Prior to the establishment of the model, the following assumptions should be considered: (1) At a specific period, the bus operates along a predetermined route. (2) All bus routes are not allowed to go beyond the platform and overtake. (3) Passengers who queue up should obey the rule of "first come, first on the bus." (4) Passengers only have one way to finish their trip, and they should not wait too long to leave the stations.

\subsection{Upper-level model (Frequency optimization model)}

The upper-level model establishes the frequency optimization model with the objective function of maximizing the profit of bus operators. The model is applied by using the analytical algorithm according to the traffic flow distribution in the lower-level model.

To simplify the model, this study considers the operational cost, including fixed cost, depreciation and

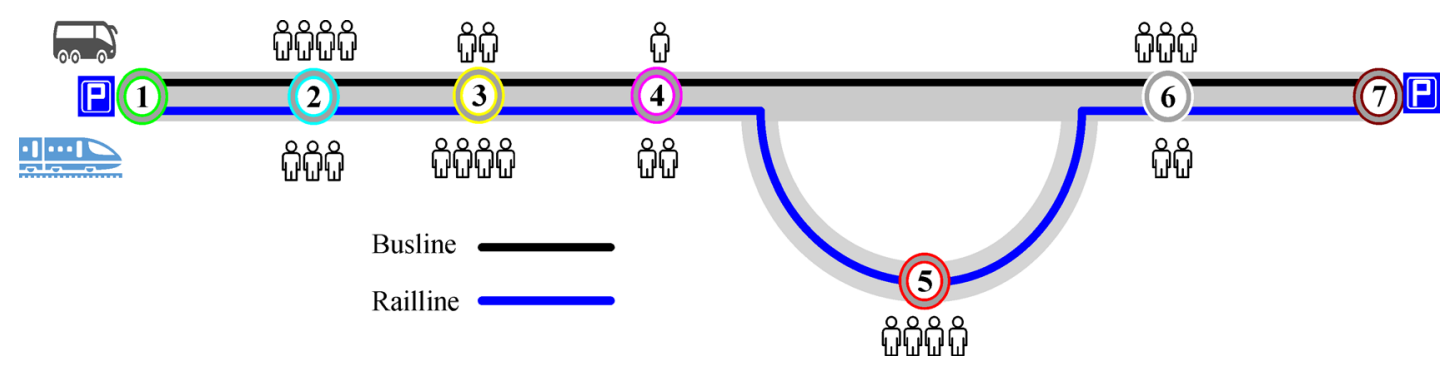

Fig. 1 Schematic of the bus and rail transit route overlap 
maintenance cost, fuel consumption, and driver and ticketing staff wages.

$$
M_{0}=C+\left(C_{c}^{v}+\lambda C_{c}^{s}\right) \times \frac{T}{h_{B}}+C_{c}^{o} \times \frac{2 \times D \times l}{h_{B}},
$$

where $M_{0}$ is the operating cost of bus operators, $T$ is the bus operating cycle, $h_{B}$ is the time interval of bus departure, $D$ is the effective operating time of buses daily, and $l$ is the distance between the starting point and terminal. $C$ denotes fixed costs, and $\lambda$ refers to the rate between drivers and vehicles for maintaining normal bus operations at 2.5 in general.

$C_{c}^{v}, C_{c}^{O}$ and $C_{c}^{S}$ refer to the cost coefficient of a vehicle, the cost coefficient of operation, and the wage coefficient of drivers and ticketing staff, respectively.

The revenue of operators is the bus ticket fares, which is shown as

$$
B_{0}=60 \times F_{B} \times \sum_{i} a_{i} \times D,
$$

where $B_{0}$ is the revenue of bus operators, and $a_{i}$ is the arrival rate of passenger $i$.

In conclusion, the integrated objective function of the upper-level model is shown as

$$
\max Z=B_{0}-M_{0} \text {. }
$$

\subsection{Lower-level model (Traffic mode split model)}

The lower-level model is based on agent simulation, in which Binomial-Logit model is used to divide different passengers in different traffic modes. When passengers are in a non-common route station, they only have one traffic mode (bus or rail transit) available. When passengers are in a common route station, they have two traffic modes to choose from. As passengers may make a comprehensive judgment on the basis of past travel experiences, such as interval of the departure time an congestion of a bus, passenger preferences would also vary. Some passengers prefer a low-cost mode, whereas others prefer a more comfortable means of transport. Furthermore, if the frequency of the bus operation becomes high, which means that that extra waiting time will not be long, then the passengers who would opt for the rail transit may reelect to the bus.

On the basis of the above analysis, the factors of travel time, fare, and congestion are considered to establish a Binomial-Logit model to analyze the probability of the chosen traffic modes of bus and rail transit.

According to random utility theory, different options will produce utility for passengers. Passengers will choose programs with the largest utility using their cognition under specific conditions. In this study, Binomial-Logit model is used to describe passenger behavior. The general form of the Binomial-Logit model is

$$
P_{m}=\frac{e^{V_{m}}}{\sum_{j \in A} e^{V_{m}}},
$$

where $P_{m}$ is the probability for the passengers to choose plan $m ; V_{m}$ is the utility for the passengers to choose plan $m$; and $A$ is the set of plans for the passengers to choose from. $X_{k}$ is the linear relationship between factors and feature vectors. Utility function is

$$
V_{m}=b_{k}+\beta_{k} X_{k},
$$

where $b_{k}$ is the constant that denotes the variable that cannot be expressed specifically in the formula, and $\beta_{k}$ is the unknown parameter of $k$ that corresponds to the characteristic variable. The factors that affect the traffic mode selection of passengers are shown in Table 1.

Table 1 Factors affecting the traffic mode selection of passengers

\begin{tabular}{lcc}
\hline Factors & Variable & Constant \\
\hline Travel time & $X_{1}$ & \\
Travel fare & $X_{2}$ & \\
Congestion & $X_{3}$ & \\
Other factors & & $b_{k}$ \\
\hline
\end{tabular}

We then quantify the factors influencing the traffic mode selection of passengers.

(1) Travel time. Assume that only one way of transport is chosen during a trip. Travel time refers to time taken to finish the entire trip, including the time spent walking to the station, waiting time, and the travel time inside the bus.

The travel time for passengers to complete a trip by bus can be expressed as Eq. (6).

$$
T_{B}=\frac{W_{B}}{v_{b}}+H_{B}+\frac{L-W_{B}}{v_{B}},
$$

where $W_{B}$ is the walking distance for passengers by bus; $v_{b}$ is the speed of walking; $L$ is the walking distance for passengers; $v_{B}$ is the speed of the bus; $H_{B}$ is the waiting time of passengers for the bus, and the waiting time is impacted by bus frequency. According to queuing theory, the average waiting time can be expressed by half of the bus departure time interval, which is $H_{B}=\frac{h_{B}}{2}$ (Dubois et al., 1979).

The travel time for the passengers to finish a trip by rail transit can be expressed as Eq. (7).

$$
T_{R}=\frac{W_{R}}{v_{b}}+H_{R}+O_{R}+\frac{L-W_{R}}{v_{R}} .
$$

where $W_{R}$ is the walking distance for passengers by rail; $v_{R}$ is the speed of the rail; and $H_{R}$ is the waiting time of passengers for the rail. The average waiting time that can be taken as half of the rail departure time interval (Guan, 
2004) is $H_{R}=\frac{h_{R}}{2}$; and $O_{R}$ is the time for passengers to access the rail platform. $O_{R}=q_{1}+\frac{k q_{2}}{v_{b}}$, where $q_{1}$ is the horizontal distance, $q_{2}$ is the vertical elevation difference, and $k$ is the coefficient between going up and down stairs. The value of going up stairs is 4.0, the value of getting down stairs is 2.0 , and the value of taking an escalator is 1.0 .

(2) Travel fare. The travel fare for passengers to achieve a trip by bus can be expressed as Eq. (8).

$$
F_{B}=k_{B}+f_{B} \times\left(L-W_{B}-L_{B}\right),
$$

where $F_{B}$ is the fare for traveling by bus, $k_{B}$ is the basic bus fare, and $f_{B}$ is the bus fare level. If the fare policy uses an integrated ticketing system, then $f_{B}$ is equal to $0 . L_{B}$ is the travel distance that covers basic bus fare.

The travel fare for passengers to achieve a trip by rail transit can be expressed as Eq. (9).

$$
F_{R}=k_{R}+f_{R} \times\left(L-W_{R}-L_{R}\right),
$$

where $F_{R}$ is the fare for traveling by rail, $k_{R}$ is the basic rail fare, and $f_{R}$ is the level for the rail fare. If the fare policy uses an integrated ticketing system, then $f_{R}$ is equal to $0 . L_{R}$ is the travel distance that covers basic rail fare.

(3) Congestion. The congestion cost for passengers to complete a trip by bus and rail transit can be expressed as Eqs. (10) and (11).

$$
\begin{gathered}
Y_{B}=\frac{\left(\sum_{i=1}^{n}\left(Z_{B u}^{i}-Z_{B d}^{i}\right)-S_{B}\right)}{C_{B}} ; \\
Y_{R}=\frac{\left(\sum_{i=1}^{n}\left(Z_{R u}^{i}-Z_{R d}^{i}\right)-S_{R}\right)}{C_{R}},
\end{gathered}
$$

where $Z_{B u}^{i}$ and $Z_{B d}^{i}$ are the number of passengers getting on and off at station $i$, respectively. $S_{B}$ refers to the number of bus seats. $C_{B}$ is the bus capacity. The rail transit notations are the same.

Capacity constraints are as follows:

$$
\begin{aligned}
& \sum_{i=1}^{n}\left(Z_{B u}^{i}-Z_{B d}^{i}\right) \leqslant C_{B} ; \\
& \sum_{i=1}^{n}\left(Z_{R u}^{i}-Z_{R d}^{i}\right) \leqslant C_{R} .
\end{aligned}
$$

(4) Other factors. As the factor of reliability and safety are difficult to quantify, a fixed parameter $b$ based on previous research is used to represent the factor in this study.

The specific formulas are as follows:

$$
\begin{gathered}
P_{B}=\frac{e^{-\theta V_{B}}}{e^{-\theta V_{B}}+e^{-\theta V_{R}}}=\frac{1}{1+e^{\left(-\theta\left(V_{R}-V_{B}\right)\right)}} ; \\
P_{R}=1-P_{B}=\frac{1}{1+e^{\left(-\theta\left(V_{B}-V_{R}\right)\right)}} ; \\
V_{B}=\beta_{1} F_{B}+\beta_{2} T_{B}+\beta_{3} Y_{B}+b_{1} ; \\
V_{R}=\beta_{1} F_{R}+\beta_{2} T_{R}+\beta_{3} Y_{R}+b_{2} .
\end{gathered}
$$

In these formulas, $P_{B}$ is the probability of choosing bus, $P_{R}$ is the probability to choosing rail transit, and $\theta$ is the sensitivity for passengers to choose a traffic mode based on the cost. $\beta_{1}, \beta_{2}, \beta_{3}$ are the coefficients of the main factors. $b_{1}, b_{2}$ are the judgment parameters for the other factors of a bus.

\section{Solution algorithm}

\subsection{SCE-UA}

Bus frequency optimization model (Eq. (3)) is a large-scale nonlinear optimization model, which addresses a complicated problem that traditional analytical methods cannot solve. Many recent studies have proven that heuristic techniques can successfully solve such complex problems (Yao et al., 2016; Yao et al., 2017; Yao et al., 2015; Yu et al., 2015), which GA, HJA, and ACO are commonly used to address. In addition, many scholars have attempted to use SCE-UA. The SCE-UA method is a novel global optimization strategy designed to be effective and efficient for a broad class of problems. This method combines the strengths of the simplex procedure by Nelder and Mead (1965) with the concepts of controlled random search (Price, 1987), competitive evolution (Holland, 1975), and complex shuffling, which is newly developed $(\mathrm{Qu}$ et al., 2008). Thus, the current paper introduces SCE-UA, which has been successfully applied in the field of water resource optimization (Duan et al., 1994).

The SCE-UA strategy procedure is presented below.

Step 1. Initialize the numbers of complexes and points in each complex as represented by $P$ and $m$, respectively. The total sample size $s=p m$.

Step 2. Generate $s$ points $x_{1}, \ldots x_{s}$ in the feasible space $\Omega \in \mathbb{R}^{n}$. Correspondingly, calculate the function value $f_{i}$ of each point $x_{i}$.

Step 3. Rank the $s$ points in ascending function value $f_{i}$. Store them in Array $D=\left\{x_{i}, f_{i}, i=1, \ldots, s\right\}$. Thus, $i=s$ represents the point with the largest function value.

Step 4. Divide $D$ into $P$ complexes $A^{1}, \ldots, A^{p}$, each with $m$ points, so that $A^{k}=\left\{x_{j}^{k} f_{j}^{k} \mid x_{j}^{k}=x_{k+p(j-1)}, f_{j}^{k}=f_{k+p(j-1)}\right.$, $j=1, \ldots, m\}$.

Step 5. Individually evolve each complex $A^{k}, k=1, \ldots, p$ on the basis of the competitive complex evolution 
algorithm.

Step 6. Replace $A^{1}, \ldots, A^{p}$ into $D$ to shuffle the complexes, such that $D=\left\{A^{k}, k=1, \ldots, p\right\}$. Sort $D$ in order of increasing function value.

Step 7. Check for convergence. If the convergence criteria are satisfied, then stop; otherwise, return to Step 4.

In Step 5, the complex evolution algorithm is used to evolve each complex. The detailed process of the complex evolution algorithm is as follows:

Step 5.1 Initialize the parameters $q, \sigma$, and $\tau$, where, $q$ is the number of subcomplexes, $\sigma$ is the target number of generations, and $\tau$ is the target number of evolutions of each complex. Note that $2 \leqslant \mathrm{q} \leqslant \mathrm{m}, \sigma \geqslant 1$, and $\tau \geqslant 1$.

Step 5.2 Use a trapezoidal probability distribution to produce weights in $A^{k}$, that is, the weight of $x_{1}^{k}$ is

$$
\rho_{i}=\frac{2(m+1-i)}{m(m+1)}, i=1, \ldots, m .
$$

Point $x_{1}^{k}$ has the highest probability $\rho=2 / m+1$. Point $x_{m}^{k}$ has the lowest probability $\rho_{m}=2 / m(m+1)$.

Step 5.3 Randomly choose $q$ distinct points $u_{1}, \ldots, u_{q}$ from $A^{k}$ according to the probability distribution specified above. Store them in Array $B=\left\{u_{i}, v_{i}, i=1, \ldots, q\right\}$, where $v_{j}$ is the function value of point $u_{j}$. Store the relative location of $u_{j}$ of $A^{k}$ in $L$.

Step 5.4 Generate offspring according to the following procedure:

(a) Sort $B$ and $L$ so that the $q$ points are arranged in ascending order of function value and then use the formula to calculate centroid $g$ :

$$
g=\frac{1}{q-1} \sum_{j=1}^{q-1} u_{j}
$$

(b) Compute the new point $r=2 g-u_{q}$ (reflection step). (c) If $r$ is within the feasible space $\Omega$, compute the function value $f_{r}$, and proceed to Step d; otherwise, compute the smallest hypercube $H \in \mathbb{R}^{n}$ that contains $A^{k}$, randomly generate a point $z$ within $H$, compute $f_{z}$, set $r=z$, and set $f_{r}$ $=f_{z}$ (mutation step). (d) If $f_{r}<f_{q}$, then replace $u_{q}$ by $r$, and proceed to Step f; otherwise, compute $c=\left(g+u_{q}\right) / 2$ and $f_{c}$ (contraction step). (e) If $f_{c}<f_{q}$, then replace $u_{q}$ by $c$, and proceed to Step f; otherwise, randomly generate a point $z$ within $H$ and compute for $f_{z}$ (mutation step). Replace $u_{q}$ by z. (f) Repeat Steps a-e $\sigma$ times, where $\sigma \geqslant 1$ is a userspecified parameter.

Step 5.5 Replace parents by offspring as follows: Replace $B$ into $A^{k}$ using the original location stored in $L$. Sort $A^{k}$ in order of increasing function value.

Step 5.6 Iterate by repeating Steps $2-5 \tau$ times, where $\tau \geqslant 1$ is a user-specified parameter which determines how many offspring should be generated (how far each complex should evolve).
The SCE-UA method version used for optimization in this study uses the values $m=(2 n+1), q=(n+1), \sigma=1$, and $\tau=(2 n+1)$. These parameter values in SCE-UA are presented Table 2 . Hence, the only variable to be specified by the user is the number of complexes $p$.

Table 2 Parameters in SCE-UA

\begin{tabular}{lllll}
\hline$m$ & $p$ & $q$ & $\sigma$ & $\tau$ \\
\hline 21 & 4 & 11 & 1 & 21 \\
\hline
\end{tabular}

To clarify the methodology and clearly indicate the novelty of the solution procedure, the algorithm flowchart of SCE-UA method is shown in Fig. 2.

\subsection{Agent simulation}

The lower-level model is the traffic mode split by using a Binomial-Logit model based on agent simulation (Gershenson, 2001). Many agent simulation software are currently available worldwide. The current study uses a NetLogo platform for the simulation (Pursula, 1999). NetLogo is utilized to simulate a social phenomenon as it can design and model an environment, and it is suitable for the system simulation of evolution over time. Model developers give instructions to the hundreds of independent operation bodies (agents). An agent enables a microcosmic-level exploration between individual behavior and macro models. The macroscopic model is composed of many individual interactions (Erol et al., 2000).

The model building process includes initialization and operation phases. Initialization creates a $2 \mathrm{D}$ simulation of the bus operating environment, including route and waiting areas for bus passengers. Bus and rail agents are produced on the route area, and the actual uncertain bus frequency is simulated. The simulation can depict the operating environment of bus frequency, including traffic jams and waiting at crossroads. According to the set rule, buses have different departure time intervals, whereas rail transit has a set departure time interval. In each station, passengers in the waiting area should appoint a passenger management agent. A management agent can produce a certain number of passenger agents in a certain period. Thus, the arrival rate of passenger is fixed. The passenger agent observes the rules of their utility function during operations. When bus and rail agents arrive in the waiting area, passengers will choose whether to get on or not.

\section{Case study}

To test the practicability and reliability of the model and algorithm, this paper takes the data of the $\mathrm{D}-\mathrm{K}$ bus route and Light Rail Route No.3 as examples. The above model 


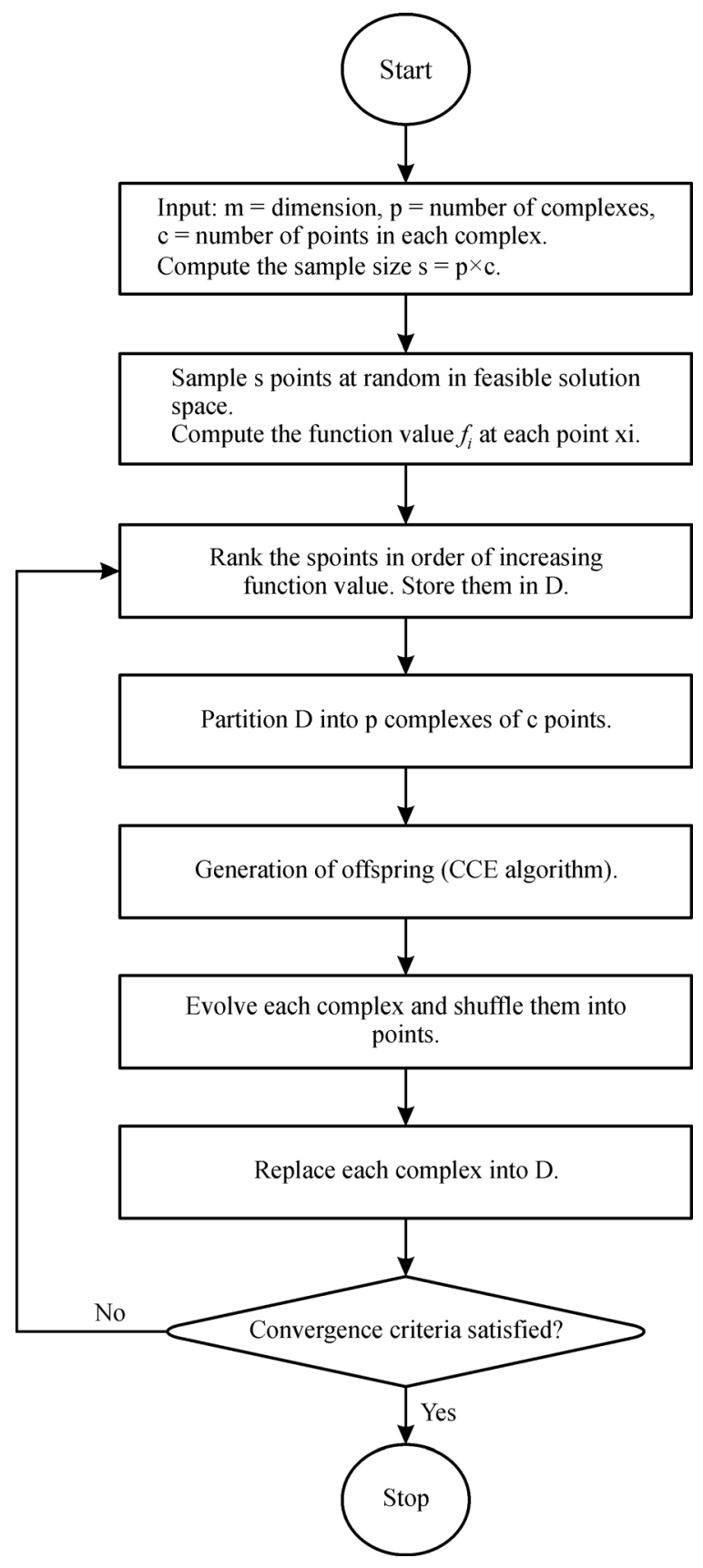

Fig. 2 Flowchart of SCE-UA

and algorithm are used to achieve optimal bus frequency.

According to the reality, Light Rail Route No. 3 and D$\mathrm{K}$ bus route overlapped in several stations from the Dalian to the Kaifaqu station. The overlapped stations of Light Rail Route No. 3 are Dalian, Xianglujiao, Jinjiajie, Quanshui, Houyan, Dalianwan, and Jinmalu stations (Fig. 3). The corresponding overlapped stations of D-K bus route are Dalian, Xianglujiao, Jinsanjiao, Ocean Natural, Distribution Market, No. 68 Middle School, and Jinmalu stations (Fig. 4).

On the basis of the investigation and statistics on Dalian, several assumptions for the model are drawn as follows:
(1) When passengers are in an overlapped station, 30\% and $20 \%$ of them are fixed passengers for bus and rail transit, respectively, that is, fixed passengers will not change their choices even if the condition changes.

(2) Assume that the rest of the passengers, except for fixed passengers, can choose their means of finishing the trip according to individual utility in an overlapped station.

(3) The last two overlapped stations will not be considered in ascending and descending of the bus and light rail routes. In other words, the last two overlapped stations are stable.

\subsection{Relevant parameters}

To complete the questionnaire relevant to passengers' flow of $\mathrm{D}-\mathrm{K}$ route with revealed preference (RP) and stated preference (SP), the questionnaire was handed out in bus stations, which are near subway stations, during the waiting time of passengers. Basic information about passengers and travel information of the RP data, as well as fare, travel distance, and travel intention of SP data are obtained.

The travel data of D-K bus and Light Rail No. 3 present the average rate data of passengers' arrival for $\mathrm{D}-\mathrm{K}$ route and Light Rail No. 3 (person/min) with the distribution of the passengers. Data are shown in Table 3.

This study aims to optimize bus frequency considering the overlapped stations of Light Rail No. 3 and D-K bus. Table 4 presents the passengers' arrival rates of Light Rail Route No. 3 at each overlapped station.

On the basis of the bus condition of Dalian city, determine $v_{b}=4.4 \mathrm{~km} \cdot \mathrm{h}^{-1}, \quad v_{B}=18 \mathrm{~km} \cdot \mathrm{h}^{-1}, v_{R}=$ $56.1 \mathrm{~km} \cdot \mathrm{h}^{-1}, H_{R}=1 / 30 \mathrm{~h}$, and $O_{R}=1 / 10 \mathrm{~h}$. Public transport fares in Dalian city are shown in Table 5. Calibration values are calculated by least squares calibration. The values are as follows: $\theta=0.9, b_{1}=-0.3, b_{2}=$ - 0.2. After establishing the basic model of passengers for choosing bus or rail transit, the values of walking distance (W) should be determined. The probability of their choice for bus and rail transit can be determined. According to the survey data, the average distances are 600 and $1500 \mathrm{~m}$ for passengers from starting point to bus and rail transit stations, respectively. Thus, the walking distance parameters for bus and rail transit are determined at 0.6 and 1.5 $\mathrm{km}$, respectively.

The parameter values of the frequency optimization model are shown in Table 6.

\subsection{Calculation results}

Set the departure time interval of Light Rail Route No. 3 as $10 \mathrm{~min}$. Change the frequency of the $\mathrm{D}-\mathrm{K}$ bus route, and substitute the parameter values in Eqs. (14) and (15). The sharing ratios of D-K bus route and Light Rail Route No. 3 under different bus frequencies are obtained, as shown in Fig. 5. 


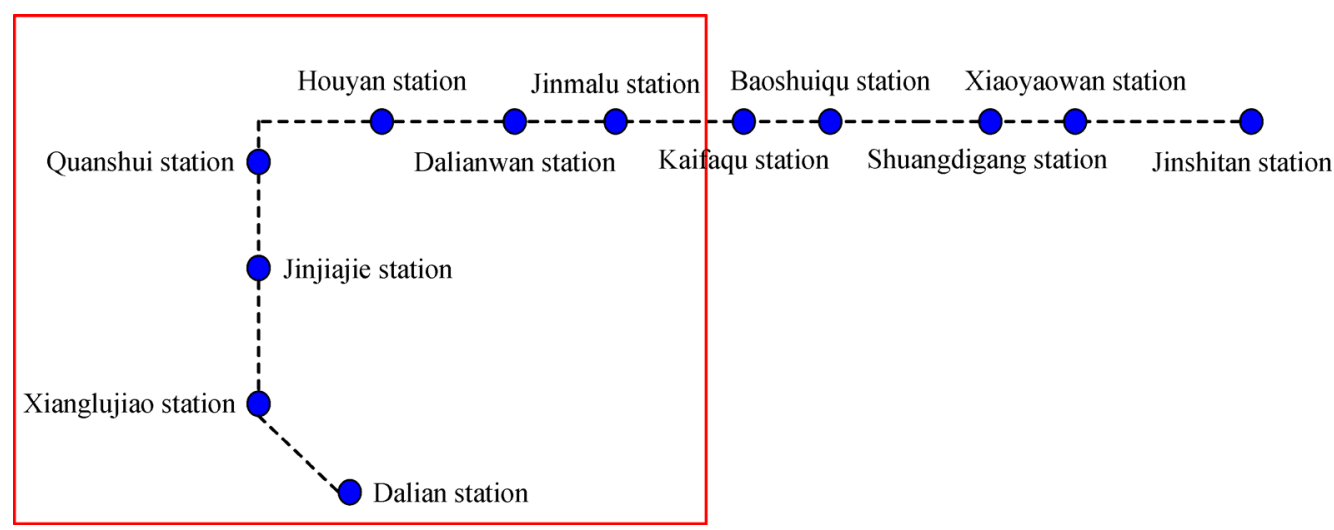

Fig. 3 Overlapped stations of Light Rail Route No. 3
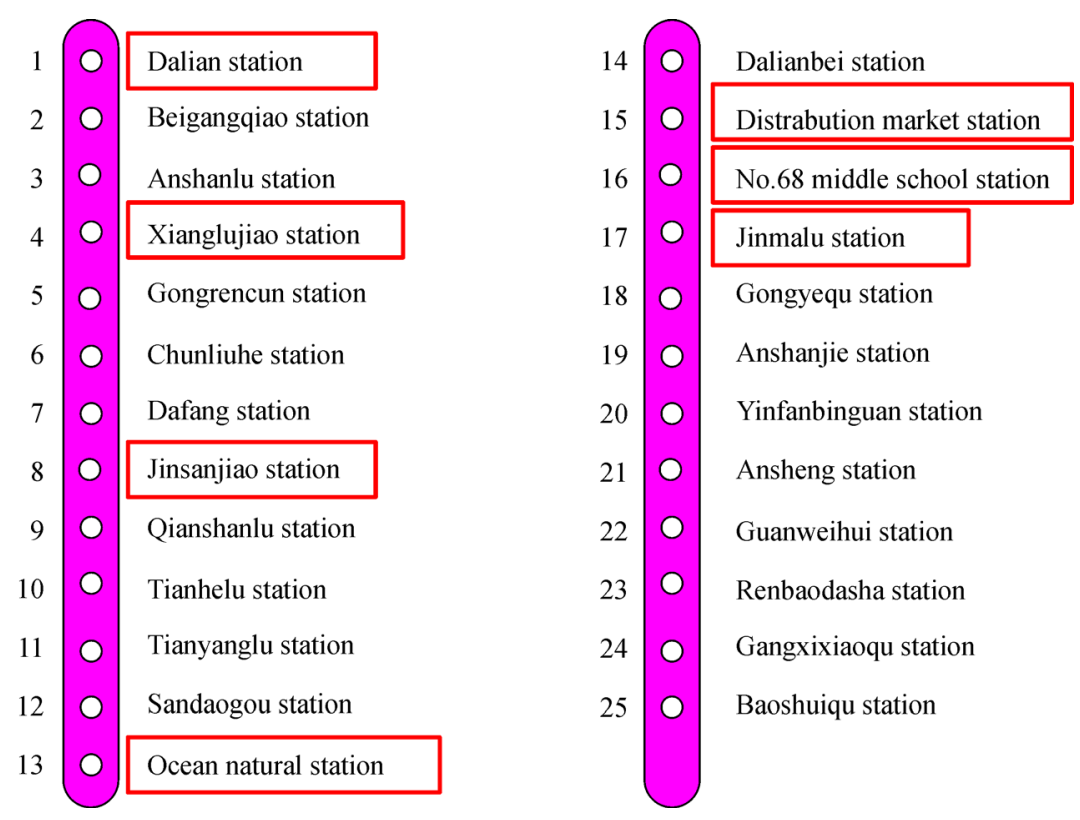

Fig. 4 Overlapped stations of D-K bus

Table 3 Passenger's arrival rate in the D-K route stations

\begin{tabular}{lc||lc}
\hline Station & Passengers' arrival rate & Station & Passengers' arrival rate \\
\hline 1 & 14.07 & 14 & 6.38 \\
2 & 8.52 & 15 & 5.23 \\
3 & 15.08 & 16 & 5.36 \\
4 & 13.27 & 17 & 6.47 \\
5 & 15.97 & 18 & 3.28 \\
6 & 5.78 & 19 & 4.05 \\
7 & 8.67 & 20 & 3.1 \\
8 & 9.43 & 21 & 1.52 \\
9 & 4.83 & 22 & 0.25 \\
10 & 6.7 & 23 & 0.13 \\
11 & 3.8 & 24 & 0.03 \\
12 & 7.78 & 25 & 0 \\
13 & 4.9 & & \\
\hline
\end{tabular}

Public sharing rate is used in the upper-level model according to the arrival rate of a bus and the number of passengers choosing the bus on each station. The profit of bus operators per vehicle every day under different bus frequencies is then obtained. Results are shown in Fig. 6.

Table 4 Passengers' arrival rates of Light Rail Route No. 3 at each overlapped station

\begin{tabular}{lc}
\hline Station & Passengers' arrival rate \\
\hline 1 & 28.33 \\
2 & 17.28 \\
3 & 18.62 \\
4 & 14.91 \\
5 & 10.4 \\
6 & 10.13 \\
7 & 15.58 \\
\hline
\end{tabular}


Table 5 Two types of public transport fares

\begin{tabular}{lcc}
\hline Public transport mode & Base price/CNY & Increasing ticket policy \\
\hline D-K route & 2 & Before $10 \mathrm{~km}$, fare will start at 2 CNY and increase every $4 \mathrm{~km}$ by $0.5 \mathrm{CNY}$; ticket prices up to $3 \mathrm{CNY}$ \\
$\begin{array}{l}\text { Light Rail Route No. } 3 \text { of } \\
\text { Dalian }\end{array}$ & 5 & Before $4.1 \mathrm{~km}$, fare will start at 2 CNY and increase every $4.4 \mathrm{~km}$ by $1 \mathrm{CNY}$ until the fare reaches 10 \\
\hline
\end{tabular}

Table 6 Parameter values of frequency optimization model

\begin{tabular}{lccc}
\hline$T$ & $D$ & $C_{c}^{v}$ & $C_{c}^{o}$ \\
\hline $7200 \mathrm{~s}$ & $16 \mathrm{~h}$ & $128 \mathrm{CNY} \cdot \mathrm{d}^{-1}$ per vehicle & $1.6 \mathrm{CNY} \cdot \mathrm{km}^{-1}$ \\
$C_{c}^{s}$ & $C$ & $l$ & $\lambda$ \\
$280 \mathrm{CNY} \cdot \mathrm{d}^{-1}$ & $1000 \mathrm{CNY} \cdot \mathrm{d}^{-1}$ & $27.4 \mathrm{~km}$ & 2.5 \\
\hline
\end{tabular}

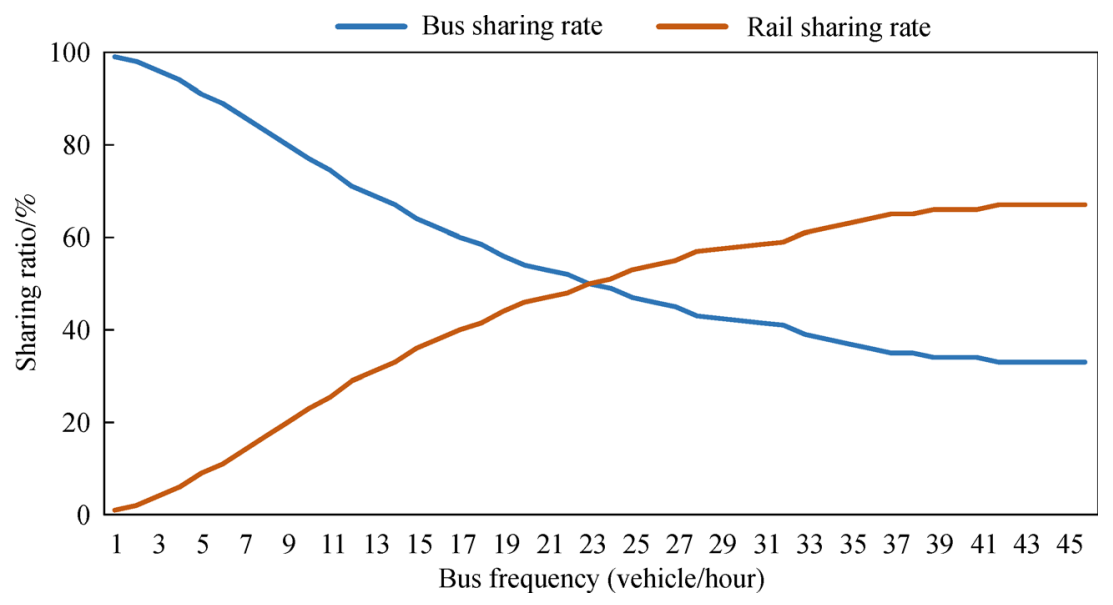

Fig. 5 Sharing ratios of D-K bus route and Light Rail Route No. 3 under different bus frequencies

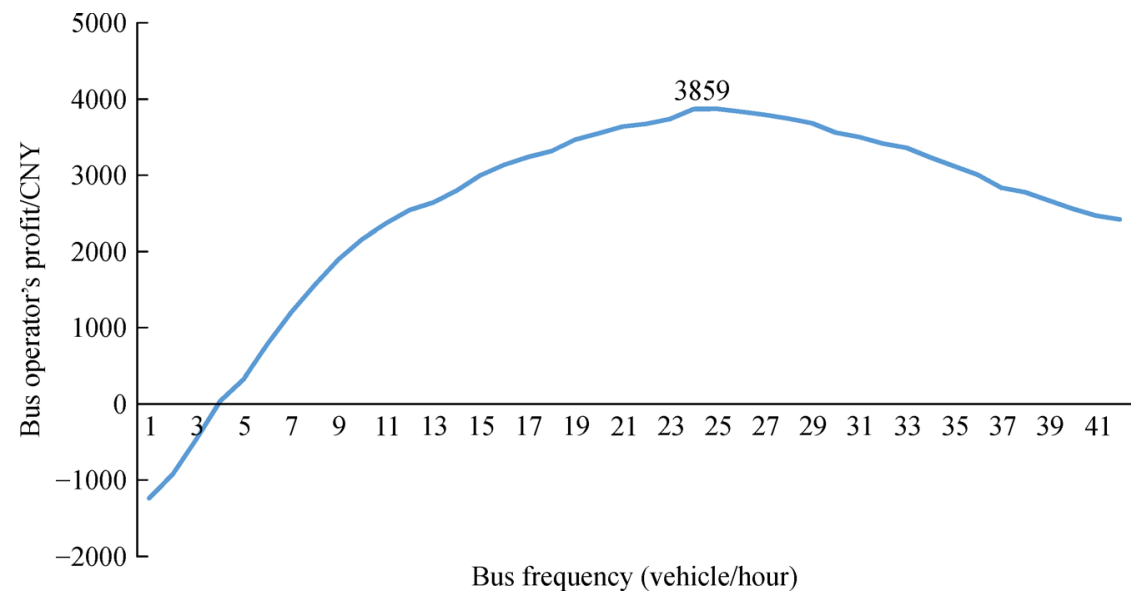

Fig. 6 Bus operator's profit under different bus frequencies 
As shown in Fig. 6 , the biggest profit is $3859 \mathrm{CNY} \cdot \mathrm{d}^{-1}$ when bus frequency is 25 per hour. When bus frequency is less than 25 per hour, the profit of bus operators will increase constantly with the increase of the bus sharing rate. By contrast, when the bus frequency is more than 25 per hour, the profit will decrease with the increase of the bus sharing rate. This condition can be attributed to the fact that passenger revenue is insufficient to sustain additional bus operating costs when sharing rate increases slowly. For managers, they should keep the bus frequency at 25 per hour and attempt to balance the sharing ratio of bus and light rail in order to maximize profit.

Finally, the paper discusses the results and analyzes the validity of the model. Under the environment of clusters (window platform), the model is solved by simulation, whereas the algorithm is solved by MATLAB 2013. The $\mathrm{D}-\mathrm{K}$ route frequency is maintained at 25 per hour. Test results are obtained after similar calculations for 20 times are shown in Table 7.

As shown in Table 7, uncertain simulations result in 10 different profit results. The mean absolute percentage error (MAPE) is calculated, which is only $3.17 \%$. The MAPE is satisfactory, and the final result is valid.

\subsection{Convergence test}

In the upper-level model, the bus frequencies of the algorithms of GA, HJA, ACO, and SCE-UA with the increase of generation are shown in Fig. 7.

As shown in Fig. 7, with the increase of generation, bus frequency continuously decreases and eventually stabilizes at 25. Thus, all four methods can satisfy the convergence test, which are feasible. However, SCE-UA achieves the optimal solution by only 80 iterations, which has the fastest convergence speed. Therefore, compared with other commonly used optimization algorithms, SCE-UA is a heuristic algorithm that can efficiently solve the upperlevel model and is evidently feasible and superior.

\section{Conclusions}

A bi-level model is used in this study to obtain optimal bus frequency. In the upper-level model, the frequency optimization model is established, and a heuristic algorithm called SCE-UA is used to solve it. The lower-level model is a Logit model based on agent simulation for traffic mode split. To analyze the variation of sharing ratio between rail transport and bus, this study establishes the competition model of bus and rail transport. As the bus headway increases, the share ratio of bus gradually increases, and the share ratio of rail transport gradually decreases. The law is also influenced by the fare system and the passengers' walking distance to the station. Passengers can choose their traffic mode by calculating their utility. Final results show that when the bus headway comes to 25 per hour, the profit of bus operators is maximized. The profit will decrease as the bus headway increases further because the growth rate of the sharing ratio gradually slows down. SCE-UA is evidently feasible

Table 7 Results of 10-time tests on bus operator's profit with 25 per hour bus frequency

\begin{tabular}{lccccc}
\hline Test times & 1 & 2 & 3 & 4 & 3 \\
\hline Bus operator's profit/CNY & 3859 & 3835 & 3866 & 3853 & 3901 \\
Test times & 6 & 7 & 8 & 9 & 10 \\
Bus operator's profit/CNY & 3872 & 3850 & 3861 & 3881 & 3927 \\
\hline
\end{tabular}

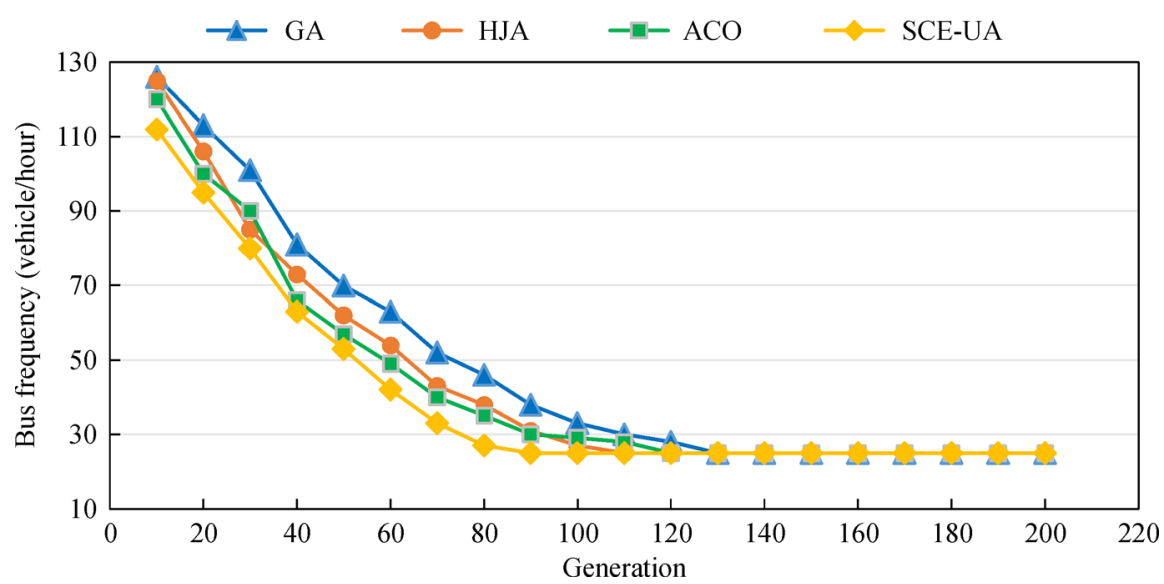

Fig. 7 Bus frequencies of GA, HJA, ACO, and SCE-UA 
and outperforms the three other optimization algorithms. SCE-UA is an efficient heuristic algorithm for solving the upper-level model in this study.

\section{References}

Agrawal J, Mathew T V (2004). Transit route network design using parallel genetic algorithm. Journal of Computing in Civil Engineering, 18(3): 248-256

Baaj M H, Mahmassani H S (1995). Hybrid route generation heuristic algorithm for the design of transit networks. Transportation Research Part C: Emerging Technologies, 3(1): 31-50

Ben-Akiva M, Morikawa T (2002). Comparing ridership attraction of rail and bus. Transport Policy, 9(2): 107-116

Blum J J, Mathew T V (2011). Intelligent agent optimization of urban bus transit system design. Journal of Computing in Civil Engineering, 25(5): 357-369

Chakroborty P (2003). Genetic algorithms for optimal urban transit network design. Computer-Aided Civil and Infrastructure Engineering, 18(3): 184-200

Chakroborty P, Deb K, Sharma R K (2001). Optimal fleet size distribution and scheduling of transit systems using genetic algorithms. Transportation Planning and Technology, 24(3): 209225

Chakroborty P, Deb K, Subrahmanyam P S (1995). Optimal scheduling of urban transit systems using genetic algorithms. Journal of Transportation Engineering, 121(6): 544-553

Constantin I, Florian M (1995). Optimizing frequencies in a transit network: A nonlinear bi-level programming approach. International Transactions in Operational Research, 2(2): 149-164

Duan Q, Sorooshian S, Gupta V K (1992). Effective and efficient global optimization for conceptual rainfall-runoff models. Water Resources Research, 28(4): 1015-1031

Duan Q, Sorooshian S, Gupta V K (1994). Optimal use of the SCE-UA global optimization method for calibrating watershed models. Journal of Hydrology, 158(3-4): 265-284

Duan Q Y, Gupta V K, Sorooshian S (1993). Shuffled complex evolution approach for effective and efficient global minimization. Journal of Optimization Theory and Applications, 76(3): 501-521

Dubois D, Bel G, Llibre M (1979). A set of methods in transportation network synthesis and analysis. Journal of the Operational Research Society, 30(9): 797-808

Erol K R, Levy R, Wentworth J (2000). Application of agent technology to traffic simulation. Strategic Planning, 1-5

Furth P G, Wilson N H M (1981). Setting frequencies on bus routes: Theory and practice. Transportation Research Record: Journal of the Transportation Research Board, (818): 1-7

Gershenson C (2001). Artificial societies of intelligent agents. Fundacion Arturo Rosenblueth Unpublished Thesis

González-Savignat M (2004). Will the high-speed train compete against the private vehicle. Transport Reviews, 24(3): 293-316

Guan H Z (2004). Disaggregate Model-Traffic Behavior Analysis Tools. Beijing: China Communications Press, 12: 1-5

Han A F, Wilson N H M (1982). The allocation of buses in heavily utilized networks with overlapping routes. Transportation Research Part B: Methodological, 16(3): 221-232

Holland J (1975). Adaptation in natural and artificial systems: An introductory analysis with applications to biology, control and artificial intelligence. Quarterly Review of Biology, 6(2): 126-137

Kuan S N, Ong H L, Ng K M (2006). Solving the feeder bus network design problem by genetic algorithms and ant colony optimization. Advances in Engineering Software, 37(6): 351-359

Lam W H K, Cheung C Y, Poon Y F (1999). A study of passenger discomfort measures at the Hong Kong mass transit railway system. Journal of Advanced Transportation, 33(3): 389-399

LeBlanc L J (1988). Transit system network design. Transportation Research Part B: Methodological, 22(5): 383-390

Mandel B, Gaudry M, Rothengatter W (1992). Linear or nonlinear utility functions in logit models? The impact on german high-speed rail demand forecasts. Transportation Research Part B: Methodological, 28B(2): 91-101

Mohaymany A S, Gholami A (2010). Multimodal feeder network design problem: Ant colony optimization approach. Journal of Transportation Engineering, 136(4): 323-331

Nakagawa D, Hatoko M (2007). Reevaluation of Japanese high-speed rail construction: Recent situation of the north corridor Shinkansen and its way to completion. Transport Policy, 14(2): 150-164

Nelder J A, Mead R (1965). A simplex method for function minimization. Computer Journal, 7(4): 308-313

Nijkamp P, Reggiani A, Tritapepe T (1996). Modelling inter-urban transport flows in Italy: A comparison between neural network analysis and logit analysis. Transportation Research Part C: Emerging Technologies, 4(6): 323-338

Nuzzolo A, Crisalli U, Gangemi F (2000). A behavioural choice model for the evaluation of railway supply and pricing policies. Transportation Research Part A: Policy and Practice, 34(5): 395-404

Park S J (2005). Bus network scheduling with genetic algorithms and simulation. Dissertation for the Master Degree. Maryland: University of Maryland

Price W L (1987). Global optimization algorithms for a CAD workstation. Journal of Optimization Theory and Applications, 55 (1): 133-146

Pursula M (1999). Simulation of traffic systems-an overview. Journal of Geographic Information and Decision Analysis, 3(1): 1-8

Qu J, Jin Q L, Xu B Y (2008). Parameter identification theory of a complex model based on global optimization method. Science in China Series G: Physics. Mechanics and Astronomy, 51(11): 17221732

Salzborn F J M (1972). Optimum bus scheduling. Transportation Science, 6(2): 137-148

Salzborn F J M (1980). Scheduling bus systems with interchanges. Transportation Science, 14(3): 211-231

Tom V M, Mohan S (2003). Transit route network design using frequency coded genetic algorithm. Journal of Transportation Engineering, 129(2): 186-195

Verma A, Dhingra S L (2006). Developing integrated schedules for urban rail and feeder bus operation. Journal of Urban Planning and Development, 132(3): 138-146

Yao B Z, Chen C, Cao Q, Jin L, Zhang M, Zhu H, Yu B (2017). Shortterm traffic speed prediction for an urban corridor. Computer-Aided 
Civil and Infrastructure Engineering, 32(2): 154-169

Yao B Z, Hu P, Lu X H, Gao J, Zhang M (2014). Transit network design based on travel time reliability. Transportation Research Part C: Emerging Technologies, 43: 233-248

Yao B Z, Hu P, Yu L, Zhang M, Gao J (2015). Merged automobile maintenance part delivery problem using an improved artificial bee colony algorithm. Scientia Iranica, 22(3): 1258-1270

Yao B Z, Yu B, Hu P, Gao J, Zhang M (2016). An improved particle swarm optimization for carton heterogeneous vehicle routing problem with a collection depot. Annals of Operations Research,
242(2): 303-320

Yu B, Kong L, Sun Y, Yao B, Gao Z (2015). A bi-level programming for bus lane network design. Transportation Research Part C: Emerging Technologies, 55: 310-327

Yu B, Lam W H K, Tam M L (2011). Bus arrival time prediction at bus stop with multiple routes. Transportation Research Part C: Emerging Technologies, 19(6): 1157-1170

Yu B, Yang Z, Yao J (2010). Genetic algorithm for bus frequency optimization. Journal of Transportation Engineering, 136(6): 576583 